\title{
Validity and repeatability of a simple index derived from the short physical activity questionnaire used in the European Prospective Investigation into Cancer and Nutrition (EPIC) study
}

\author{
Nicholas J Wareham ${ }^{1, *}$, Rupert W Jakes ${ }^{1}$, Kirsten L Rennie ${ }^{1}$, Jantine Schuit ${ }^{2}$, Jo Mitchell ${ }^{1}$, \\ Susie Hennings ${ }^{1}$ and Nicholas E Day ${ }^{1}$ \\ 'Department of Public Health and Primary Care, Institute of Public Health, University of Cambridge, Robinson Way, \\ Cambridge CB2 2SR, UK: ${ }^{2}$ Department of Chronic Disease and Environmental Epidemiology, \\ National Institute of Public Health and the Environment, Bilthoven, The Netherlands
}

Submitted 10 June 2002: Accepted 24 October 2002

\begin{abstract}
Objective: To assess the validity and repeatability of a simple index designed to rank participants according to their energy expenditure estimated by self-report, by comparison with objectively measured energy expenditure assessed by heart-rate monitoring with individual calibration.

Design: Energy expenditure was assessed over one year by four separate episodes of 4-day heart-rate monitoring, a method previously validated against whole-body calorimetry and doubly labelled water. Cardio-respiratory fitness was assessed by four repeated measures of sub-maximum oxygen uptake. At the end of the 12-month period, participants completed a physical activity questionnaire that assessed pastyear activity. A simple four-level physical activity index was derived by combining occupational physical activity together with time participating in cycling and other physical exercise (such as keep fit, aerobics, swimming and jogging).

Subjects: One hundred and seventy-three randomly selected men and women aged 40 to 65 years.

Results: The repeatability of the physical activity index was high (weighted kappa $=0.6, P<0.0001)$. There were positive associations between the physical activity index from the questionnaire and the objective measures of the ratio of daytime energy expenditure to resting metabolic rate $(P=0.003)$ and cardiorespiratory fitness $(P=0.001)$. As an indirect test of validity, there was a positive association between the physical activity index and the ratio of energy intake, assessed by 7-day food diaries, to predicted basal metabolic rate.

Conclusions: The summary index of physical activity derived from the questions used in the European Prospective Investigation into Cancer and Nutrition (EPIC) study suggest it is useful for ranking participants in terms of their physical activity in large epidemiological studies. The index is simple and easy to comprehend, which may make it suitable for situations that require a concise, global index of activity.
\end{abstract}

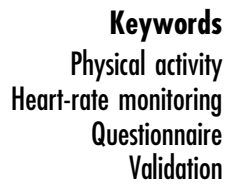

Keywords Validation
The European Prospective Investigation into Cancer and Nutrition (EPIC) was started in 1989 as a multi-centre prospective cohort study of the link between diet and the risk of developing cancer at a range of sites ${ }^{1}$. This large study combines cohorts in nine different European countries and has recruited a total of 455751 individuals who have been studied according to a common protocol ${ }^{2}$. The measurement of physical activity is important in this cohort for a number of reasons. Physical activity is a major potential confounding factor in any study of the relationship between dietary factors and chronic disease. It is also an important exposure in its own right, and the EPIC study presents an ideal opportunity to investigate the links between activity and chronic disease. This is particularly true of cancer, for which the epidemiological links to physical activity remain to be more firmly established $^{3}$. One advantage of this study is that the large sample size will result in a sufficient number of cases for site-specific analyses to be undertaken.

The EPIC baseline examination included questions on physical activity that were derived from development work undertaken in The Netherlands. A longer form of this EPIC physical activity questionnaire has previously been compared with a 3-day activity diary ${ }^{4}$. This study concluded that the questionnaire satisfactorily ranked participants according to their physical activity but was not 
suitable for estimating energy expenditure. However, a study that attempts to validate a measurement instrument against another of the same fundamental type runs the risk of being affected by correlated error ${ }^{5}$. The current study was designed to compare the EPIC physical activity questionnaire with objective measures of cardio-respiratory fitness and energy expenditure assessed by heart-rate monitoring with individual calibration, measures that have themselves been validated against gold standard techniques and which are unlikely to have correlated error with a questionnaire. The repeatability of the questionnaire is also described. In addition to analysing the EPIC questions in the manner that was previously described by Pols et al. ${ }^{4}$, we also compared a new four-point physical activity index derived from the same questions. This simple index was constructed from the EPIC questionnaire in order to combine reported participation in occupational and recreational activity.

\section{Methods}

\section{The EPIC physical activity questions}

The four EPIC physical activity questions (see Appendix) refer to activity during the past year. The first question is a four-point, mutually exclusive, ordered category concerning physical activity at work. The second question asks about the amount of time spent in hours per week for summer and winter separately in each of the following activities: walking, cycling, gardening, do-it-yourself, physical exercise and housework. The original description of the EPIC questions suggested calculation of reported energy expenditure in all of the activities in question 2 by multiplying reported time by standard energy costs from published compendia ${ }^{6}$. The third question asks whether any of the activities in question 2 were engaged in such that it caused sweating or faster heartbeat and, if so, for how many hours during a typical week. The fourth question asks about stair climbing.

As occupational activity and recreational activity are both likely to be relevant to total energy expenditure, we devised a simple physical activity index to allocate individuals to ordered categories of overall activity. Our primary concern was that this index should remain as close to the data as possible and should have high face validity. A priori we decided to allocate individuals who did not report occupational activity to the sedentary group. The simple four-level classification of self-reported occupational activity and four-level categorisation of time spent in cycling and other physical exercise were combined to form a physical activity index. We restricted our attention only to cycling and other physical exercise because, in general, higher-intensity physical activities are reported with greater accuracy in physical activity questionnaires $^{7}$. The original $4 \times 4$ matrix of occupational and recreational activity would have contained too many cells to be useful in an epidemiological study and each cell would have contained too few individuals for the validation study to make any definitive statements. Therefore we condensed the 16 groups into four categories with the intention that within each category activity levels would be comparable and that the population would be roughly evenly distributed across the groups.

\section{Validity study}

The study was conducted in a subgroup of volunteers who were selected at random from a continuing populationbased cohort study in Ely, Cambridgeshire, the detailed design of which has been described previously ${ }^{8,9}$. Briefly, the original sample of 1122 individuals without known diabetes was recruited between 1990 and 1992 at random from a population-based sampling frame consisting of all people in Ely, Cambridgeshire aged between 40 and 65 years in age in $1990^{10}$. The initial response rate was $74 \%$. Between 1994 and 1997 a 4.5-year follow-up study was undertaken of all those individuals who did not have diabetes, according to World Health Organization criteria, at baseline $(n=1071)$. A random subset of 200 individuals from this cohort was asked to re-attend a further three times at 3-month intervals over the following year when all tests were repeated. One hundred and seventy-three of the participants completed all measurements and therefore had four measures of cardio-respiratory fitness and four measures of 4-day energy expenditure by heart-rate monitoring completed across 1 year (see Fig. 1). At the final visit, participants completed the physical activity questionnaire that refers to activity in the past 12 months.

On each of the four visits, height and weight were measured in light clothing and body fat percentage was obtained using a standard impedance technique (Bodystat, Isle of Man). Body circumferences were measured using a metal tape. The waist circumference was measured at the mid-point between the lower costal margin and the level of the anterior superior iliac crest. Hip circumference was measured at the level of the greater trochanter. The protocol for undertaking the individual calibration between heart rate and energy expenditure has been reported previously ${ }^{9,11}$. The oxygen consumption-heart

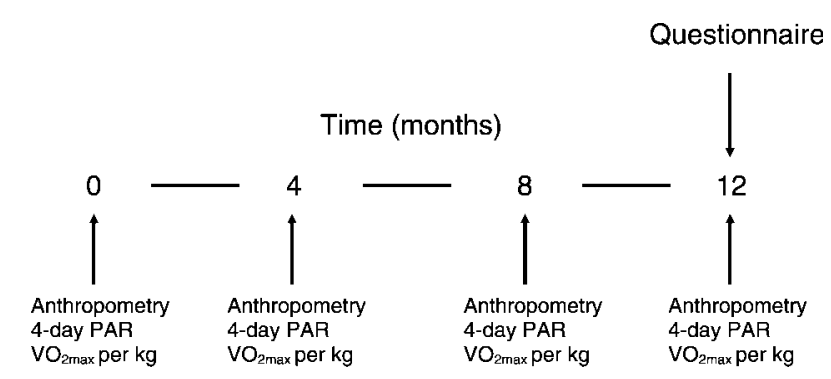

Fig. 1 Time intervals for the assessment of objective measures of physical activity and assessment of past-year physical activity by the questionnaire. PAR - physical activity ratio 
rate relationship was assessed at rest with the subject lying and then seated, using an oxygen analyser calibrated daily using 100\% nitrogen and fresh air as standard gases. To provide the slope and the intercept of the line relating energy expenditure to heart rate, each participant cycled at $50 \mathrm{rev} \mathrm{min}^{-1}$ and the workload was increased progressively from $0 \mathrm{~W}$, through $37.5 \mathrm{~W}, 75 \mathrm{~W}$ and $125 \mathrm{~W}$ in stages, each lasting $5 \mathrm{~min}$. At each workload three separate readings were made of heart rate, minute volume and oxygen concentration of expired air. The $125 \mathrm{~W}$ level was only undertaken if the heart rate had not reached 120 beats per minute by the end of the $5 \mathrm{~min}$ at $75 \mathrm{~W}$. The oxygen concentration in the expired air and minute volume data were used to calculate oxygen consumption after correction for standard temperature and pressure. Energy expenditure $\left(\mathrm{kJ} \mathrm{min}^{-1}\right)$ was calculated ${ }^{12}$ at each time point as oxygen consumption $\left(\mathrm{ml} \mathrm{min}^{-1}\right) \times 20.35$. Mean resting energy expenditure was taken as the average of the lying and sitting values. Flex heart rate, the empirical point at which the distinction between rest and exercise is made, was calculated as the mean of the highest resting pulse rate and the lowest on exercise. Finally, the slope and intercept of the least-squares regression line of the exercise points were calculated. Maximum oxygen uptake $\left(\mathrm{VO}_{2 \max }\right)$ was measured from the linear regression as predicted oxygen consumption at maximal heart rate $(220$ - age) and is expressed in the results per unit body weight. The volunteers wore the heart-rate monitor (Polar Electro, Finland) continuously during the waking hours over the following four days. Heart-rate readings were downloaded directly into a computer via a serial interface and the individual calibration data were used to predict minute energy expenditure for each person. The energy expenditure data were summed over the day to create an estimate of daytime energy expenditure. This is expressed in the analysis as a physical activity ratio (PAR), calculated as the ratio of daytime energy expenditure to resting energy expenditure. For each individual, the means of body mass index, percentage body fat, waist-to-hip ratio, physical activity ratio and $\mathrm{VO}_{2 \max }$ on the four occasions were calculated and are used in the analysis as the measures of the usual level of obesity and its regional distribution, energy expenditure and fitness, respectively. As an additional but indirect test of validity, we compared the physical activity index with estimates of the ratio of energy intake to predicted basal metabolic rate $^{13}$ from 7-day food diaries that were available in 5847 participants of the EPIC-Norfolk cohort itself. Ethical permission for the study was granted by the Cambridge Local Research Ethics Committee.

\section{Repeatability study}

The repeatability of the questionnaire was assessed in a sample of participants who were recruited to the EPICNorfolk cohort ${ }^{2}$. The questionnaire was administered at baseline and then again in the follow-up questionnaire
18-21 months later $(n=2271)$. The question regarding time spent doing housework and do-it-yourself in the baseline questionnaire was not asked separately for summer and winter as it was at the follow-up questionnaire. The follow-up questionnaire did not include the question regarding stair climbing. The repeatability was assessed by calculating the weighted kappa statistic for the four-category physical activity index from the baseline and follow-up questionnaires using weights defined ${ }^{14}$ as $1-[(i-j) /(k-1)]^{2}$.

\section{Results}

The 173 participants in the validation study were middleaged men and women with anthropometric features typical of those seen in adults of this age in EPIC-Norfolk and in other population-based studies. Average daytime energy expenditure as assessed by heart-rate monitoring was higher in men than in women (Table 1). Men also had a significantly higher maximum oxygen uptake.

The simple four-level occupational classification was strongly associated with daytime energy expenditure (Fig. 2) ( $P$ for trend $<0.001$ ). There was, however, no correlation between daytime energy expenditure and the time reported participating in all activities in question

Table 1 Mean (standard deviation (SD)) anthropometric and physical activity characteristics of the validation study group $(n=173)$

\begin{tabular}{|c|c|c|c|c|c|}
\hline & \multicolumn{2}{|c|}{$\begin{array}{c}\text { Men } \\
(n=84)\end{array}$} & \multicolumn{2}{|c|}{$\begin{array}{l}\text { Women } \\
(n=89)\end{array}$} & \multirow[b]{2}{*}{$P$-value } \\
\hline & Mean & SD & Mean & SD & \\
\hline Age (years) & 58.8 & 7.9 & 55.4 & 6.7 & 0.003 \\
\hline $\mathrm{BMI}\left(\mathrm{kg} \mathrm{m}^{-2}\right)$ & 26.2 & 2.7 & 25.7 & 4.4 & NS \\
\hline Body fat by impedance (\%) & 25.1 & 3.9 & 36.4 & 6.1 & $<0.001$ \\
\hline WHR & 0.96 & 0.07 & 0.78 & 0.07 & $<0.001$ \\
\hline Daytime PAR & 2.58 & 0.47 & 2.29 & 0.38 & $<0.001$ \\
\hline $\mathrm{VO}_{2 \max }\left(\mathrm{mlmin}^{-1} \mathrm{~kg}^{-1}\right)$ & 31.3 & 7.2 & 26.6 & 5.4 & $<0.001$ \\
\hline
\end{tabular}

BMI - body mass index; NS - not significant; WHR - waist-to-hip ratio; PAR - physical activity ratio.

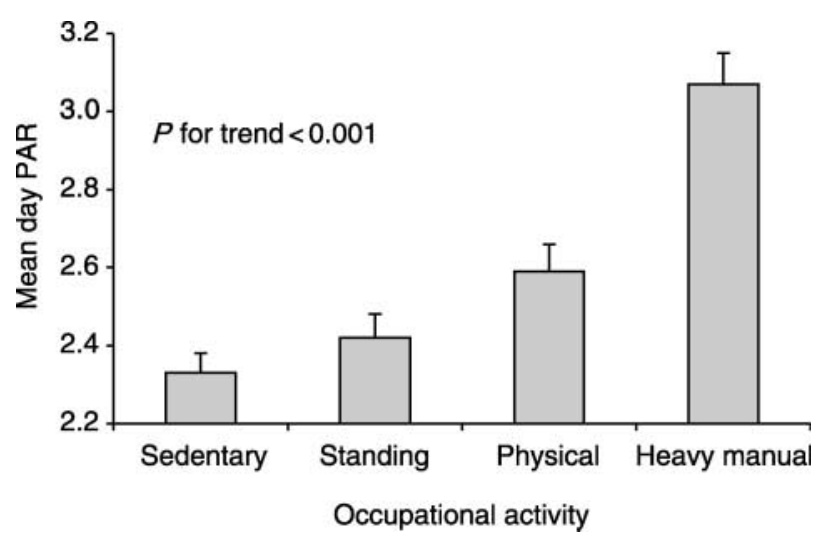

Fig. 2 Mean day physical activity ratio (PAR) by occupational physical activity $(n=173)$ 
2 combined $(r=0.04, P=0.59)$. Nor was there a correlation with reported energy expenditure $(r=0.05$, $P=0.52$ ) calculated by multiplying the amount of time spent on each activity in question 2 by the intensity of the activity, as suggested in the validation of the questionnaire by Pols and colleagues ${ }^{4}$. Eighty-five per cent of reported time for the activities in question 2 was represented by the low-intensity activities (do-it-yourself, gardening, housework and walking). Cycling and other physical exercise (such as keep fit, aerobics, swimming and jogging) contributed $15 \%$ of time reported in question 2. Figure 3 shows mean day PAR by category of reported participation in cycling and other physical exercise. Activity was

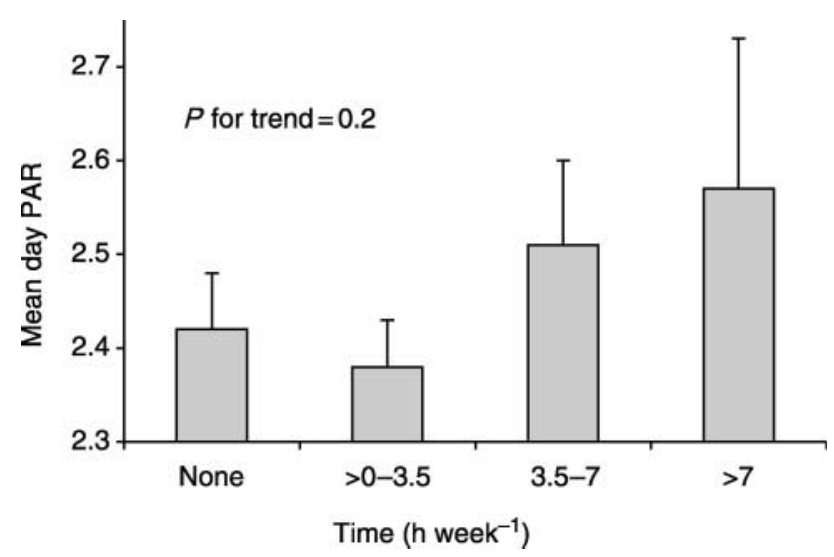

Fig. 3 Mean day physical activity ratio (PAR) by self-reported participation in cycling and other physical exercise $(n=173)$ categorised into four levels intended to have public health meaning, i.e. none, up to $0.5 \mathrm{~h} \mathrm{day}^{-1}, 0.5$ to $1 \mathrm{~h} \mathrm{day}^{-1}$ and more than 1 h day $^{-1}$. Although it did not reach conventional statistical significance, there was an apparent trend of those individuals who reported participating in more than $0.5 \mathrm{~h} \mathrm{day}^{-1}$ having higher daytime energy expenditure. A comparison of the population above and below this threshold showed that those people reporting less than $0.5 \mathrm{~h} \mathrm{day}^{-1}$ expended less energy (daytime PAR $=2.40$ ) than those who reported more than $0.5 \mathrm{~h} \mathrm{day}^{-1}$ (daytime $\mathrm{PAR}=2.53, P=0.055$ for comparison of means). There was no significant difference in mean day PAR between those who reported no bouts of more vigorous activity and those who reported some vigorous activity (question 3), nor was there any relationship between the frequency of reported stair climbing and mean day PAR (question 4).

Table 2 describes the four levels of the physical activity index and Table 3 shows the distribution of the 30410 individuals in the EPIC-Norfolk cohort into these different categories. Of all people, $30.7 \%$ were categorised as inactive, $28.7 \%$ as moderately inactive, $22.1 \%$ as moderately active and $18.5 \%$ as active. In the validation study, mean daytime energy expenditure and $\mathrm{VO}_{2 \max }$ were both positively and significantly associated with the index with and without adjustment for age and/or sex. Figures 4 and 5 illustrate the significant positive associations between mean day PAR and $\mathrm{VO}_{2 \max }$ for each category of the activity index, respectively. The analyses were repeated stratifying by sex. The overall pattern of association was similar in men and women but the significance of the relationship

Table 2 Interpretation of the physical activity index groups

\begin{tabular}{ll}
\hline Label & \multicolumn{1}{c}{ Description } \\
\hline Inactive & Sedentary job and no recreational activity \\
Moderately inactive & Sedentary job with $<0.5 \mathrm{~h}$ recreational activity per day \\
& or Standing job with no recreational activity \\
Moderately active & Sedentary job with 0.5 to $1 \mathrm{~h}$ recreational activity per day \\
& or Standing job with $0.5 \mathrm{~h}$ recreational activity per day \\
& or Physical job with no recreational activity \\
Active & Sedentary job with $>1 \mathrm{~h}$ recreational activity per day \\
& or Standing job with $>0.5 \mathrm{~h}$ recreational activity per day \\
& or Physical job with at least some recreational activity \\
& or Heavy manual job
\end{tabular}

Table 3 Matrix illustrating the frequency distribution (\%) of occupational status and reported participation in cycling and other physical exercise within EPIC-Norfolk $(n=30410)$. Symbols represent definition of physical activity index based on the distribution: *, inactive; $\dagger$, moderately inactive; $\ddagger$, moderately active; $\S$, active

\begin{tabular}{lccrr}
\hline \multirow{2}{*}{$\begin{array}{l}\text { Cycling/sports } \\
\left.\text { (h week }^{-1}\right)\end{array}$} & Sedentary & Standing & Physical & Heavy manual \\
\cline { 2 - 5 } 0 & $9335(30.7 \%)^{\star}$ & $3578(11.8 \%) \dagger$ & $2465(8.1 \%) \ddagger$ & $459(1.5 \%) \S$ \\
$0-3.5$ & $5152(16.9 \%) \dagger$ & $2526(8.3 \%) \ddagger$ & $1593(5.2 \%) \S$ & $167(0.6 \%) \S$ \\
$3.5-7$ & $1743(5.7 \%) \ddagger$ & $840(2.8 \%) \S$ & $622(2.0 \%) \S$ & $70(0.2 \%) \S$ \\
$>7$ & $936(3.1 \%) \S$ & $450(1.5 \%) \S$ & $415(1.4 \%) \S$ & $59(0.2 \%) \S$ \\
\hline
\end{tabular}




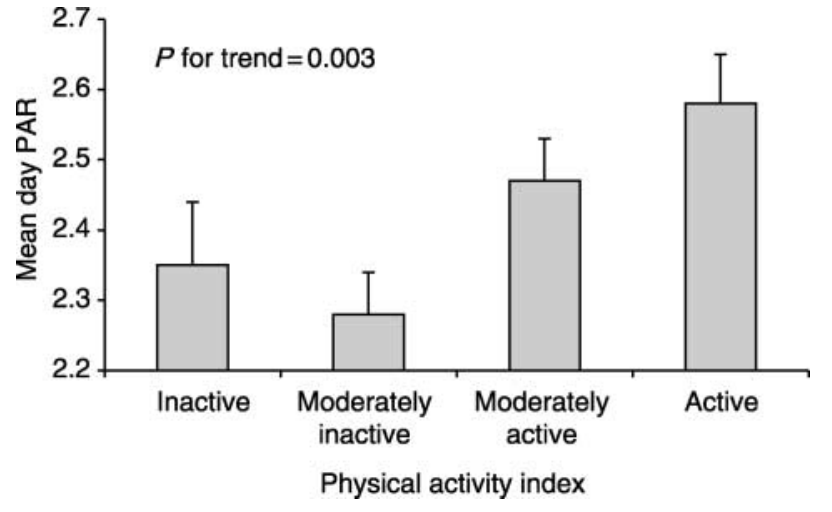

Fig. 4 Mean day physical activity ratio (PAR) by physical activity index $(n=173)$

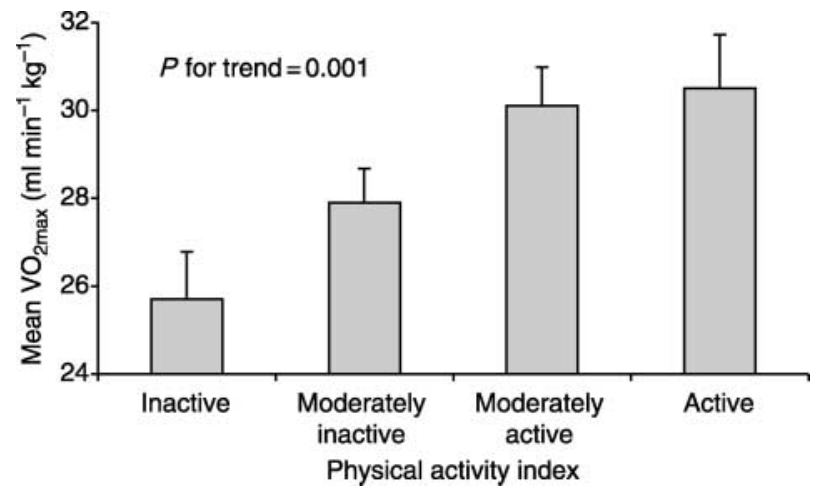

Fig. 5 Mean $\mathrm{VO}_{2 \max }$ by physical activity index $(n=173)$

between the index and the validation measures was diminished as a function of the size of the stratified samples. The comparison of the physical activity index with estimates of energy intake from 7-day food diaries in 5847 participants of the EPIC-Norfolk cohort showed a positive and statistically significant relationship in both men and women (Fig. 6).

Finally, the repeatability of the activity index was assessed by Cohen's weighted kappa statistic. Table 4 describes the matrix resulting from a comparison of

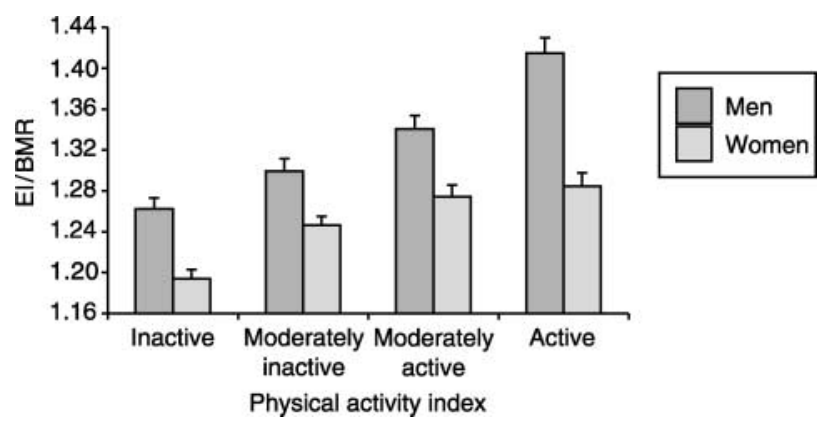

Fig. 6 Ratio of energy intake (EI), from 7-day food diary, to predicted basal metabolic rate (BMR)* by physical activity index $(n=$ 5847). *BMR calculated using prediction equations based on age, sex and weight ${ }^{13}$
Table 4 Repeatability of the physical activity index $(n=2271)$

\begin{tabular}{lrrrrr}
\hline & \multicolumn{5}{c}{ Index at follow-up } \\
\cline { 2 - 6 } Index at baseline & \multicolumn{1}{c}{1} & 2 & 3 & 4 & Total \\
\hline 1 & 512 & 119 & 48 & 22 & 701 \\
2 & 168 & 367 & 105 & 33 & 673 \\
3 & 79 & 153 & 193 & 79 & 504 \\
4 & 43 & 69 & 99 & 182 & 393 \\
Total & 802 & 708 & 445 & 316 & 2271 \\
\hline
\end{tabular}

Weighted kappa $=0.6, P<0.0001$.

baseline and a repeat assessment of the index. The weighted kappa statistic for the comparison of the physical activity index at baseline with that for the repeat survey was $0.6, P<0.0001$.

\section{Discussion}

The short EPIC physical activity questionnaire has been administered to more than 450000 participants of the EPIC study as part of the baseline health and lifestyle questionnaire. This study describes the development and validation of a physical activity index based on the short physical activity questionnaire against energy expenditure calculated from four days of heart-rate monitoring with individual calibration, repeated on four occasions throughout the time frame of the questionnaire, i.e. the past year. This is the first study, to our knowledge, that has used an objective method of measuring energy expenditure to validate this particular questionnaire.

The four-point activity index was developed primarily to rank subjects within the EPIC-Norfolk cohort according to their relative energy expenditure. Mean daytime PAR was positively associated with the four-point occupational question, although the proportion of those reporting heavy manual occupation was low. The lack of correlation of time spent in all recreational activities could be attributable to the imprecision of the assessment of low-intensity activities, which contributed to $85 \%$ of the total time reported. Although time spent participating in cycling and other physical exercise was more closely associated with energy expenditure, it did not reach conventional statistical significance. This may not be entirely surprising since it was considered in isolation from occupational activity that we had previously demonstrated to be a determinant of energy expenditure. The summary index combining both occupational and recreational activity is simple and has face validity. This study shows that the physical activity index successfully ranks participants according to their activity and cardiorespiratory fitness when assessed by objective methods. The repeatability of the derived index proved to be acceptable (weighted kappa statistic $=0.6$ ). It is likely that this is an underestimate of the repeatability of the questionnaire because of the long average time between 
completion of the baseline and repeat questionnaires in the EPIC-Norfolk cohort (18-21 months).

Few other validation studies of global indices of physical activity have been reported in the literature. The Godin Leisure-Time Exercise Questionnaire is simple and short and has been validated ${ }^{7}$ but does not include any assessment of occupational activity. The Lipid Research Clinics Physical Activity Questionnaire asks about self-perceived ranking of activity rather than absolute levels ${ }^{15}$. Most other questionnaires are much longer and are not reducible to a global index. Although in aetiological studies it may be more appropriate to employ longer physical activity questionnaires that can quantify activity in work, domestic life, travel and recreation, this is not always possible when there are constraints on the number of questions that can be included, as was the case with EPIC-Europe. A global index is also useful as a simple screen for physical activity in healthcare settings where categorisation is required to identify individuals who might benefit from more detailed assessment or targeted intervention.

The study group selected for the validation study was chosen at random from a continuing population-based cohort study and as such tends to be unselected with regard to physical activity behaviour. The participants in our validation study were on average 5 years younger than the population for whom the questionnaire was designed. However, this is unlikely to result in a biased assessment of validity, as the physical activity patterns of the two groups are likely to be similar, containing a mixture of working age and retired people.

In summary, the repeatability and validity studies on the activity index defined in this paper suggest that it is useful for ranking participants in terms of their physical activity in large epidemiological studies such as EPIC-Norfolk. As with any physical activity questionnaire and index derived from it, the inference about its validity and repeatability is limited to the population in whom it was tested (i.e. people aged 40-65 years) and its use is restricted to the purpose for which it was intended (i.e. the assessment of past-year usual activity). In other populations or age groups, different questionnaires may be more suitable. Although it was designed for the EPICEurope study, the simplicity and ease of comprehension of the short EPIC physical activity index may make it suitable for other situations where a simple global index of activity is required.

\section{Acknowledgements}

The Ely validation study was funded by a grant from the Medical Research Council (MRC) and the National Health Service Research and Development Directorate. N.J.W. was funded by an MRC Clinical Scientist Fellowship. K.L.R. and R.W.J. held MRC PhD studentships.

\section{References}

1 Riboli E. Nutrition and cancer: background and rationale of the European Prospective Investigation into Cancer and Nutrition (EPIC). Ann. Oncol. 1992; 3(10): 783-91.

2 Day N, Oakes S, Luben R, Khaw K-T, Bingham S, Welch A, et al. EPIC in Norfolk: study design and characteristics of the cohort. Br. J. Cancer 1999; 80(Suppl. 1): 95-103.

3 US Department of Health and Human Services. Physical Activity and Health: A Report of the Surgeon General. Atlanta, GA: US Department of Health and Human Services, Centers for Disease Control and Prevention, National Center for Chronic Disease Prevention and Health Promotion, 1996.

4 Pols MA, Peeters PHM, Ocke MC, Slimani N, BuenoDe-Mesquita HB, Collette HJA. Estimation of reproducibility and relative validity of the questions included in the EPIC physical activity questionnaire. Int. J. Epidemiol. 1997; 26(Suppl. 1): S181-9.

5 Wong MY, Day NE, Wareham NJ. Measurement error in epidemiology: the design of validation studies II: bivariate situation. Stat. Med. 1999; 18: 2831-45.

6 Ainsworth BE, Haskell WL, Whitt MC, Irwin ML, Swartz AM, Strath SJ, et al. Compendium of physical activities: an update of activity codes and MET intensities. Med. Sci. Sports Exerc. 2000; 32(Suppl. 9): S498-516.

7 Jacobs DR, Ainsworth BE, Hartman TJ, Leon AS. A simultaneous evaluation of 10 commonly used physical activity questionnaires. Med. Sci. Sports Exerc. 1993; 25: 81-91.

8 Wareham NJ, Byrne CD, Williams R, Day NE, Hales CN. Fasting proinsulin concentrations predict the development of type 2 diabetes. Diabetes Care 1999; 22: 262-70.

9 Wareham NJ, Wong M-Y, Day NE. Glucose intolerance and physical inactivity: the relative importance of low habitual energy expenditure and cardiorespiratory fitness. Am. J. Epidemiol. 2000; 152: 132-9.

10 Williams DRR, Wareham NJ, Brown DC, Clark PMS, Cox BD, Cox LJ, et al. Glucose intolerance in the community: the Isle of Ely Diabetes Project. Diabetic Med. 1995; 12: 30-5.

11 Wareham NJ, Hennings SJ, Prentice AM, Day NE. Feasibility of heart-rate monitoring to estimate total level and pattern of energy expenditure in a population-based epidemiological study: the Ely young cohort feasibility study 1994-5. Br. J. Nutr. 1997; 78: 889-900.

12 Elia M, Livesey G. Energy expenditure and fuel selection in biological systems: theory and practice of calculations based on indirect calorimetry and tracer methods. In: Simopoulus AP, ed. Metabolic Control of Eating, Energy Expenditure and The Bioenergetics of Obesity. Basel: Karger, 1992; 68-131.

13 James WPT, Schofield EC. Human Energy Requirements. Oxford: Oxford University Press, 1990.

14 Stata Corporation. Stata Statistical Software, Release 7.0 version [program]. College Station, TX: Stata Corporation, 2001.

15 Ainsworth BE, Jacobs DR Jr, Leon AS, Richardson MT, Montoye HJ. Assessment of the accuracy of physical activity questionnaire occupational data. J. Ocсир. Med. 1993; 35(10): 1017-27.

\section{Appendix - EPIC physical activity questions}

1. We would like to know the type and amount of physical activity involved in your work. Please tick what best corresponds to your present activities from the following four possibilities:

- Sedentary occupation 
You spend most of your time sitting (such as in an office)

- or Standing occupation

You spend most of your time standing or walking. However, your work does not require intense physical effort (e.g. shop assistant, hairdresser, guard, etc.)

- or Physical work

This involves some physical effort including handling of heavy objects and use of tools (e.g. plumber, cleaner, nurse, sports instructor, electrician, carpenter, etc.)

- or Heavy manual work

This involves very vigorous physical activity including handling of very heavy objects (e.g. docker, miner, bricklayer, construction worker, etc.)

2. In a typical week during the past 12 months, how many hours did you spend on each of the following activities? (Put '0' if none)

- Walking, including walking to work, shopping and leisure

$\begin{array}{ll}\text { in summer } & \text { hours per week } \\ \text { in winter } & \text { hours per week }\end{array}$

- Cycling, including cycling to work and during leisure time

in summer hours per week in winter hours per week

- Gardening

$\begin{array}{ll}\text { in summer } & \text { hours per week } \\ \text { in winter } & \text { hours per week }\end{array}$

- Housework such as cleaning, washing, cooking, childcare

hours per week

- Do-it-yourself

hours per week

- Other physical exercise such as keep fit, aerobics, swimming, jogging

$\begin{array}{ll}\text { in summer } & \text { hours per week } \\ \text { in winter } & \text { hours per week }\end{array}$

3. In a typical week during the past year did you practise any of these activities vigorously enough to cause sweating or a faster heartbeat?

Yes

No

Don't know

- If yes, for how many hours per week in total did you practise such vigorous physical activity? (Put ' 0 ' if none)

hours per week

4. In a typical day during the past 12 months, how many floors of stairs did you climb up? (Put '0' if none) floors per day 